\title{
Structural defects and their role in the growth of
}

\author{
Ag triangular nanoplates
}

Túlio C.R. Rocha, Daniela Zanchet

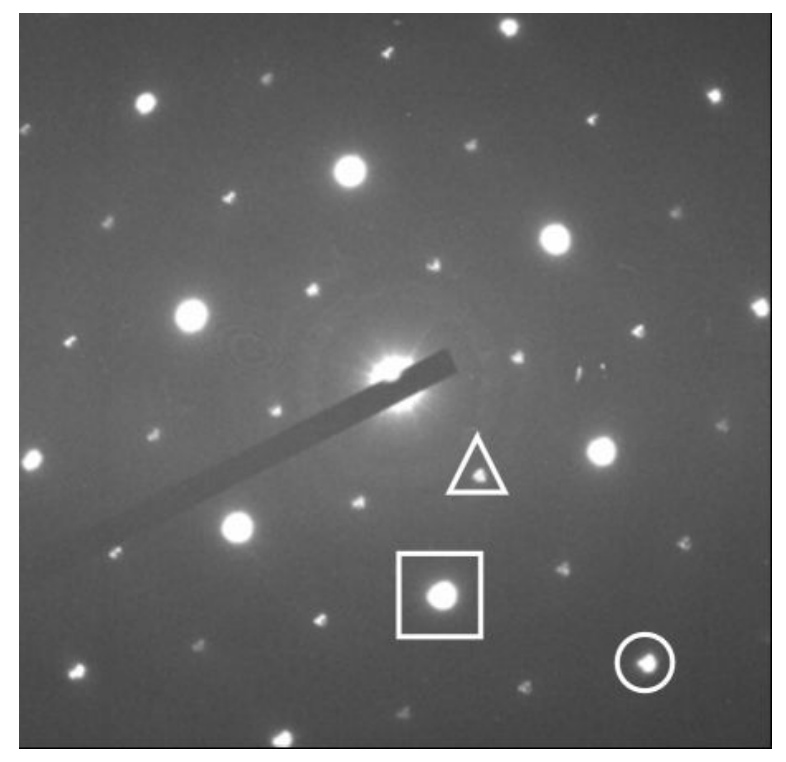

Figure S1: Selective area diffraction of a single triangular nanoplate. Marked reflections corresponds to 220 (square), 422 (circle) and $1 / 3\{422\}$ (triangle) 


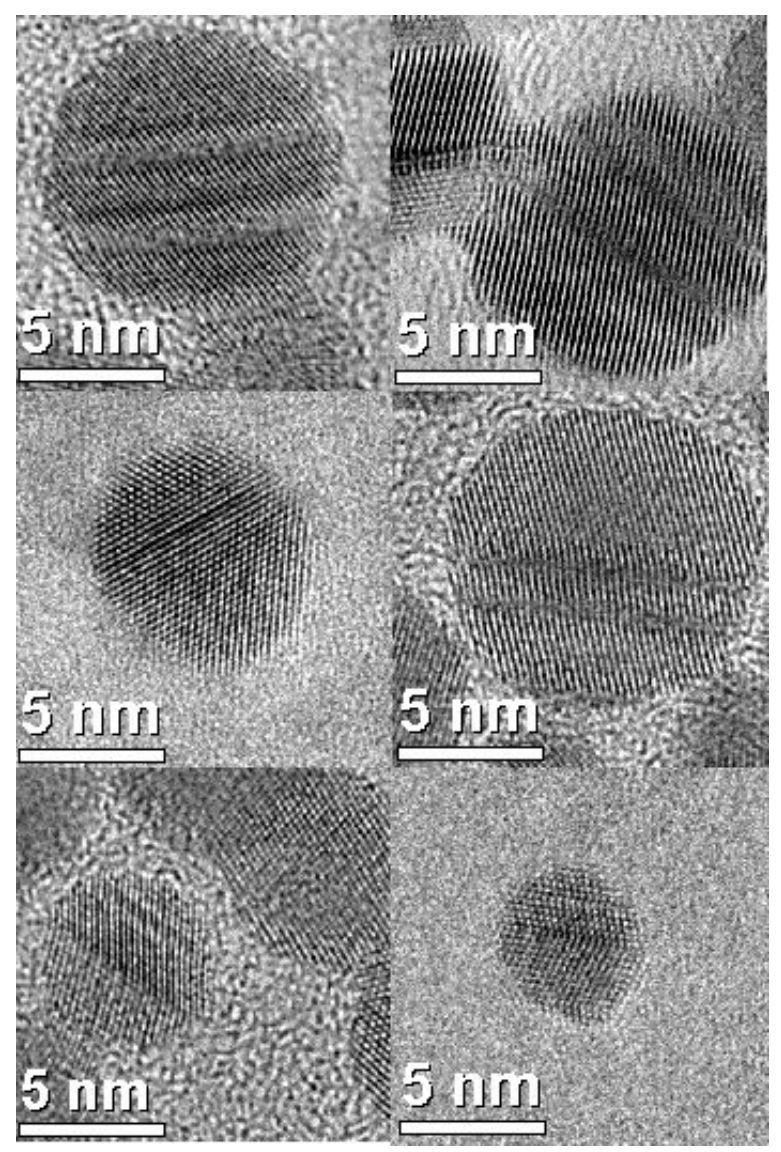

Figure S2: Ag seeds showing several planar defects 
Figure S3 recalls the schematic geometry of planar defects caused by errors in the stacking sequences of a fcc lattice. Depending on the number of planes involved, the planar defect will be called intrinsic stacking fault (isf, one plane), extrinsic stacking fault (esf, two planes) or twin, (tw, three planes or more) as shown in the figure S3-a. A twin is a mirror plane and as a consequence it creates a three layer hcp lamella inside the fcc lattice and changes crystal orientation. The hcp lamella is also produced if a stacking fault is present, because of the local mirror symmetry introduced. The local hcp structure in the vicinity of the defects are evidenced by the dotted box in figure S3-a. Figure S3-b shows the 2D projection of a fcc (left) and a hcp (right) structures viewed perpendicularly to the stacking planes. As can be seen, the local transformation of a fcc into a hcp introduces a new set of planes with $2.49 \AA$ spacing (the 1-100 hcp distance) parallel to the ordinary 422 planes.
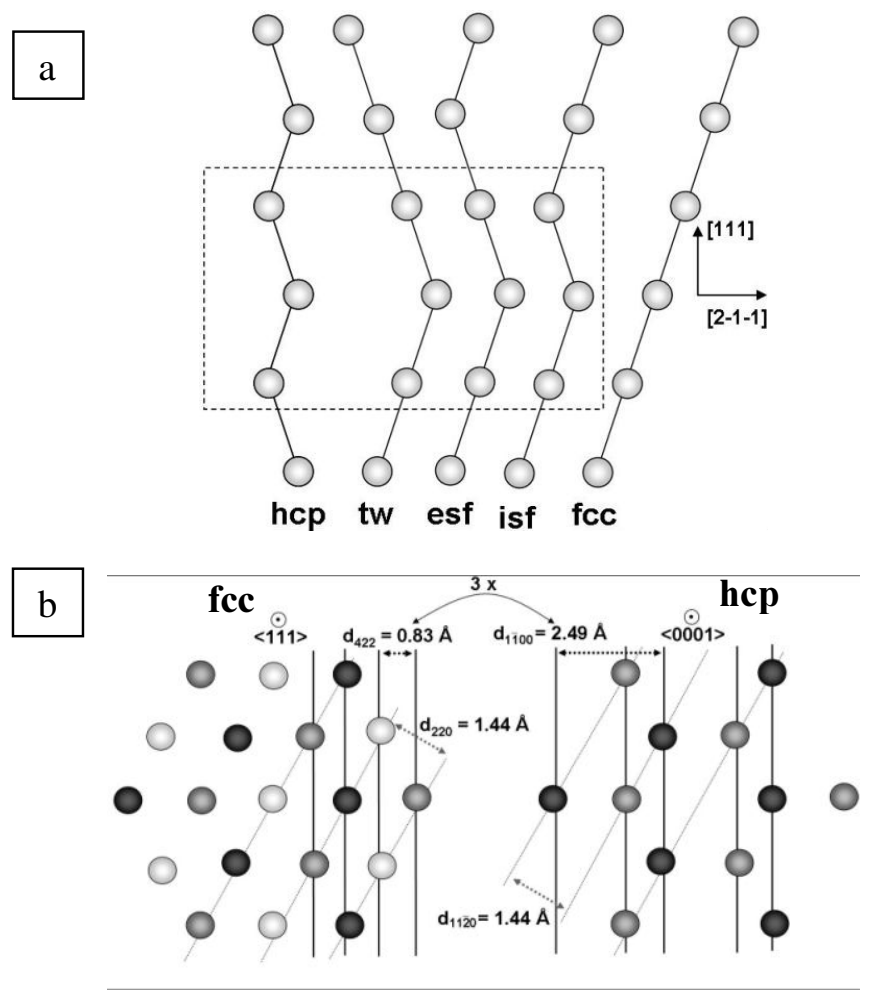

Figure S3: Schematics of the closed packed structures, fcc in the left side and hcp in the right side. (a) $<110\rangle$ (fcc) and $\langle 11-10\rangle$ (hcp) 2D projections showing the effect of planar defects with dotted box evidencing the crystallographic lamella introduced by the defects. (b) $<111>$ (fcc) and $<0001>$ (hcp) 2D projections showing the lattice plane distances. 
Figure S4 shows some diffraction patterns of individual nanoparticles obtained using the Debye equation of Kinematic diffraction. This equation calculates the scattered intensity profile for a distribution of scattering centers uniformly and randomly oriented with respect to the incident beam. This gives rise to a pattern that is a radial section of a Debye-Scherer (powder) diffraction pattern.

For a particle containing $\mathrm{N}$ atoms of one type, the Debye equation can be written as

$$
I_{N}(s)=I_{0} N f^{2}(s)\left(1+\frac{D}{N} \sum_{n \neq m}^{N} \frac{\sin \left(2 \pi s x_{n m}\right)}{2 \pi s x_{n m}}\right)
$$

Where $\mathrm{I}_{0}$ is the incident intensity and $\mathrm{I}_{\mathrm{N}}(\mathrm{s})$ is the intensity scattered per unit solid angle in the direction defined by $\mathrm{s}=2 \sin \theta / \lambda$, with $\theta$ equals to half of the scattering angle and $\lambda$ the wavelength of the $\mathrm{x}$-Rays. $\mathrm{x}_{\mathrm{nm}}$ is the distance between atom $\mathrm{n}$ and $\mathrm{m}$ in the particle. The Debye Waller factor $\mathrm{D}$, express the attenuation due to structural disorder, and it was set to 1 in this work. The scattering factor $\mathrm{f}(\mathrm{s})$ represents the single atom contribution to scattering and can be expressed using an analytic approximation by

$$
f(s)=\sum_{i=1}^{4} a_{i} e^{-b_{i}\left(\frac{s}{2}\right)^{2}}+c
$$

where ai, bi and c are coefficients available in tabulated form for most elements and radiations.

The generation of the structural models of the particles, i. e. the atomic positions, together with the evaluation of the Debye equation were implemented in a computer program using $C$ language. The single crystal structures and defective structures were obtained by filling the space with stacking planes following the desired configuration and using interatomic distances of bulk materials. Spherical particles of different size were then obtained defining a cut-off vector of the desired size. The non-crystallographic structures, decahedra and icosahedra (multiply twinned particles) were generated using the atomic coordinates prescribed by Flüeli [4], adding complete shells of atoms.

Regarding X ray or electron diffraction, the effect of the planar parallel defects in the $<111>$ direction in a fcc nanoparticle is the appearance of new reflections of an equivalent hcp structure. However, it is worth to mention that the presence of planar defects also influence the intensity and shape of the ordinary fcc reflections, since it introduces translation vectors that do not belong to the crystal space group, consequently destroying the coherence between some diffracted rays.

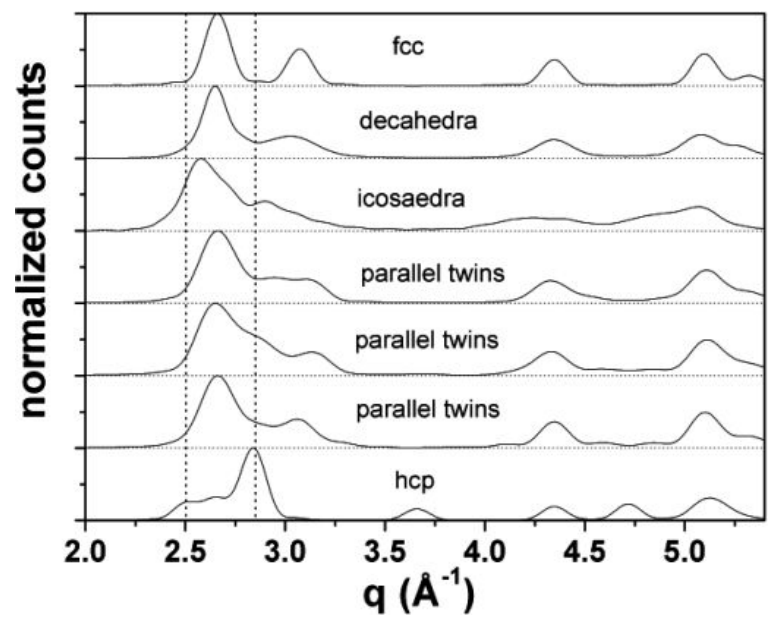

Figure S4: Theoretical diffractograms showing the appearance of hcp distances in fcc structures due to the presence of parallel planar defects. The particles in these calculations have $5 \mathrm{~nm}$ in diameter. 

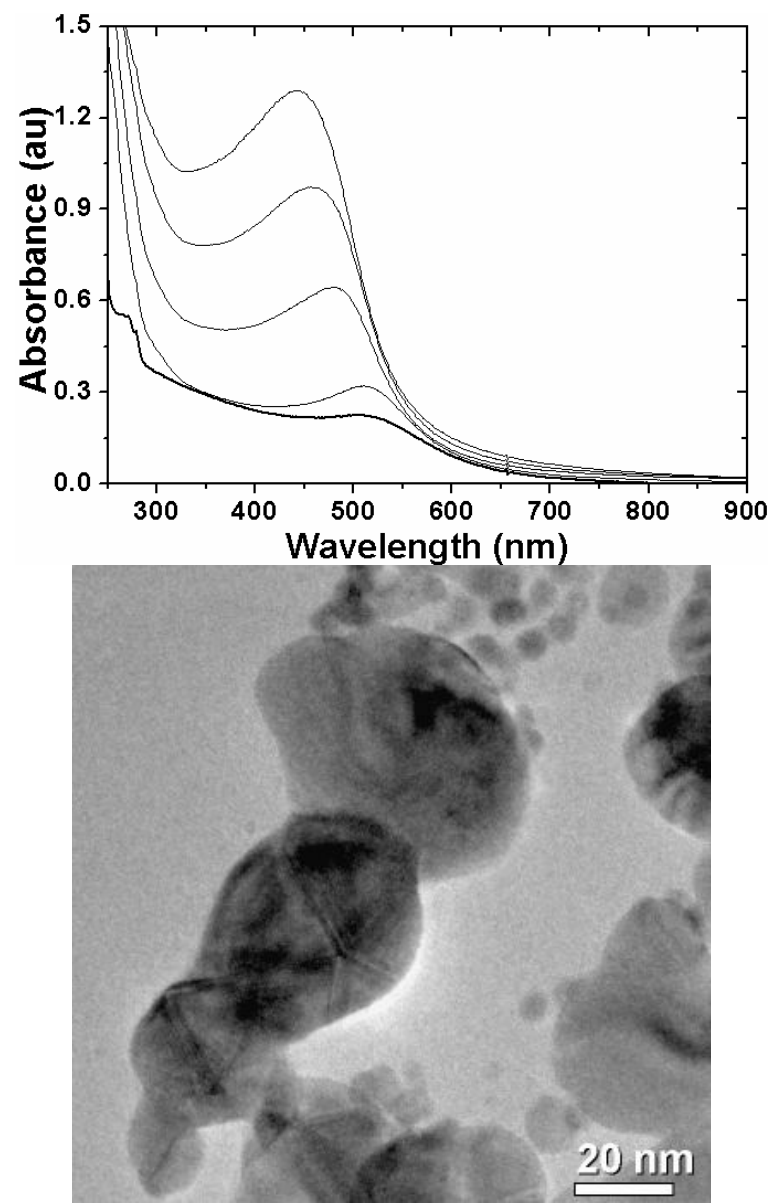

Figure S5: (a) time dependent UV- VIS during the synthesis of the Au@ Ag core-shell and (b) TEM image of large MTP and irregular particles formed after irradiation. 